\title{
Use of interfering RNA to investigate the role of endogenous gastrin in the survival of gastrointestinal cancer cells
}

\author{
AM Grabowska*,', J Hughes' and SA Watson' \\ 'Academic Unit of Cancer Studies, D Floor, West Block, Queen's Medical Centre, University Hospital, Nottingham NG7 2UH, UK
}

Gastrin isoforms, acting through a variety of receptors, have proliferative and anti-apoptotic effects on gastrointestinal (Gl) cancers. A small interfering RNA (siRNA) targeting the gastrin gene was used to investigate the role of endogenous gastrin in Gl cancer cell survival. Downregulation of the gastrin gene in siRNA-transfected cells was measured using real-time reverse transcriptase-PCR. The most effective siRNA was tested in a panel of $\mathrm{Gl}$ cancer cell lines at various concentrations and time points, and the effect on cell survival and apoptosis was measured using methyl thiazoyl tetrazolium (MTT) and caspase 3 activation assays. Gastrin siRNA reduced gene expression by more than $90 \%$ in a range of $\mathrm{Gl}$ cancer cell lines. Downregulation of the gastrin gene was dose-dependent and effective over approximately I week in vitro. However, downregulation at the protein level was delayed by 3-4 days. Gastrin siRNAtransfected cells showed up to a $60 \%$ reduction in growth and up to a $50 \%$ increase in apoptosis compared with control siRNAtransfected cells. The effects were most marked in the cell line with the highest constitutive level of gastrin gene expression (human metastatic colon, $\mathrm{CI} 70 \mathrm{HM} 2$ ) and in epidermal growth factor (EGF)-treated cells as the gastrin promoter contains an EGF-response element, gERE. The ability of the siRNAs to reduce survival of these $\mathrm{Gl}$ cell lines is further evidence of the importance of autocrine and/or intracrine gastrin loops in Gl cancer, where expression of the gastrin gene and autonomous gastrin appears widespread. British Journal of Cancer (2007) 96, 464-473. doi: I0.1038/sj.bjc.6603588 www.bjcancer.com

Published online 30 January 2007

(C) 2007 Cancer Research UK

Keywords: gastrin; gastrointestinal cancer; interfering RNA

Gastrin is produced by $\mathrm{G}$ cells in the antral mucosa, and in addition to its established role in gastric acid secretion, it plays a major role in tumorigenesis in the gastrointestinal (GI) tract. Gastrin expression is frequently increased in colonic, pancreatic and gastric adenocarcinomas compared with normal mucosa (Finley et al, 1993; Goetze et al, 2000; Henwood et al, 2001). It is expressed early in the development of GI adenocarcinomas (Smith and Watson, 2000; Henwood et al, 2001), and in an APC $^{\text {Min }}$ mouse model, hypergastrinaemia promoted the progression of premalignant lesions (Watson and Smith, 2001). Tumour growth factoralpha and epidermal growth factor (EGF) are involved in upregulation of gastrin expression in a number of colon cancer cell lines through binding to an established EGF response element, gERE, within the gastrin promoter (Howell et al, 1995; Watson et al, 2000).

In $\mathrm{G}$ cells, gastrin is processed to a 17-amino-acid amidated active product. However, in GI epithelial cells, processing is incomplete, resulting in secretion of the precursor peptide, progastrin, and a range of intermediate products, of which the most well-characterised is gly-gastrin (Rehfeld et al, 2004). Whereas amidated gastrin acts through the CCK-2 receptor (CCK-2R) (Shulkes and Baldwin, 1997), progastrin and gly-gastrin bind with low affinity to this receptor (Baldwin et al, 2001;

*Correspondence: Dr AM Grabowska;

E-mail: anna.grabowska@nottingham.ac.uk

Revised 5 December 2006; accepted 14 December 2006; published online 30 January 2007
Dockray et al, 2001). Furthermore, the biological effects of progastrin and gly-gastrin are not blocked by CCK-B receptor antagonists (Seva et al, 1994; Stepan et al, 1999; Baldwin et al, 2001), and amidated gastrin and gly-gastrin stimulate different downstream signalling events (Todisco et al, 2001). Potential alternative receptors for progastrin and gly-gastrin have been suggested (Seva et al, 1994; Baldwin, 1995a; Singh et al, 1995; Smith et al, 2002; Ahmed et al, 2004).

Exogenously applied gastrin and its precursors have a range of downstream effects on GI cancer cells. Amidated gastrin, glygastrin and progastrin promote growth of a range of cell lines in vitro and in vivo (Watson et al, 1989; Seva et al, 1994; Baldwin, 1995b; Stepan et al, 1999; Baldwin et al, 2001) and upregulate expression of a range of anti-apoptotic proteins, including Akt and bcl-2 (Konturek et al, 2003; Harris et al, 2004a; Ramamoorthy et al, 2004). Gly-gastrin promotes invasion of human colon cancer cells by upregulating expression of matrix metalloproteinases (Baba et al, 2004), by increasing the rate of metastasis formation in vivo (Kermorgant and Lehy, 2001) and by promoting blood vessel formation through the stimulation of endothelial cells (Clarke et al, 2006).

The role of endogenously produced gastrin has been investigated through the use of antibodies to gastrin and CCK-2R antagonists (Hoosein et al, 1988; Smith et al, 1996). However, such reagents may underestimate the role of gastrin, as a cocktail of reagents would be needed to inhibit all biologically active products of the gastrin gene and may be ineffective against gastrin-derived peptides that have not yet been identified. 
This study uses small interfering RNAs (siRNAs) to reduce gastrin expression in a range of GI cancer cells in order to investigate the role of endogenously produced gastrin. Gastrin antisense-transfected cell lines have previously been shown to have reduced growth in vitro and are poorly tumorigenic in vivo (Singh et al, 1996; Harris et al, 2004b; Smith et al, 2004). However, siRNAs potentially provide a more powerful and specific mechanism for the downregulation of genes than traditional antisense approaches (Aoki et al, 2003) and have stringent sequence specificity (Elbashir et al, 2001a). The data described in this paper demonstrate a protective role for endogenous gastrin in a range of GI cancer cells using an siRNA specific for gastrin.

\section{MATERIALS AND METHODS}

\section{Cell culture}

PAN-1 is a human pancreatic cell line derived from a poorly differentiated human pancreatic adenocarcinoma within the Academic Unit of Cancer Studies, University of Nottingham, UK. HCT116, a poorly differentiated human colon cell line, was obtained from ECACC (ref. no. 91091005). C170HM2 is a human colorectal tumour cell line originally derived from a poorly differentiated tumour (Watson et al, 1993). MGLVA1 is an ascitic variant of the gastric cell line, MKN 45G (Watson et al, 1990).

All cell lines were routinely cultured in RPMI 1640 culture medium (Gibco, Paisley, UK) containing 10\% (v/v) heat-inactivated fetal bovine serum (Sigma, Poole, UK) at $37^{\circ} \mathrm{C}$ in $5 \% \mathrm{CO}_{2}$ and humidified conditions.

\section{Small interfering RNA synthesis}

Target sites within the gastrin gene for siRNA synthesis were chosen for the following properties (Elbashir et al, 2001b):

(a) preceded by $\mathrm{AA}$ in the gene sequence

(b) avoidance of the extreme $5^{\prime}$ or $3^{\prime}$ ends of the gene

(c) $30-60 \%$ GC content

(d) absence of long stretches of A's

(e) lack of homology with other genes using BLAST

Their sequences are given in Table 1 . Initial experiments were carried out with siRNAs synthesised in vitro using the Silencer siRNA Construction Kit (Ambion, Austin, TX, USA), according to the manufacturer's instructions. Briefly, template DNA oligonucleotides containing the sense or antisense of the target sequence, followed by the leader sequence CCTGTCTC at the $3^{\prime}$ end, were synthesised commercially (Helena Bioscience, Gateshead, UK). The T7 promoter-containing primer was annealed to the template oligonucleotides and dsDNA created using Klenow DNA polymerase. The sense and antisense dsDNAs were used as templates to synthesise the antisense or sense strands of RNA,

Table I Gastrin siRNA target sequences

\begin{tabular}{llcc}
\hline Target & Target sequence & \% GC & $\begin{array}{c}\text { Position } \\
\text { in gene }\end{array}$ \\
\hline $\operatorname{tg} 1$ & AAGCTTCTTGGAAGCCCCGCT & 57.1 & 59 \\
$\operatorname{tg} 5$ & AAGAAGCAGGGACCATGGCTG & 57.1 & 220 \\
$\operatorname{tg} 7$ & AAGAAGAAGAAGCCTATGGAT & 38.1 & 245 \\
$\operatorname{tg} 8$ & AAGAAGAAGCCTATGGATGGA & 42.9 & 248 \\
$\operatorname{tg} 9$ & AAGAAGCCTATGGATGGATGG & 47.6 & 251 \\
$\operatorname{tg} 10$ & AAGCCTATGGATGGATGGACT & 47.6 & 254 \\
scr tg5 & AAGAGATGTAAGGCCAGGCCG & 57.1 & NA \\
scr tg8 & AAGCGAAGAAACGAGGTGTT & 42.9 & NA \\
\hline
\end{tabular}

NA: not applicable. respectively, using T7 RNA polymerase. The two RNA strands were annealed together, digested with DNase and RNase to remove the template DNA and leader sequence, then column-purified and stored at $-80^{\circ} \mathrm{C}$. The concentration of the siRNAs was calculated by measuring their optical density (OD) at $260 \mathrm{~nm}$.

Small interfering RNAs were also synthesised commercially as separate strands that were annealed and PAGE-purified (Dharmacon, Lafayette, CO, USA). A fluorescent version of tg8 was also synthesised with a TAMRA label at the $5^{\prime}$ end of the sense strand.

\section{Small interfering RNA transfection}

Cells $\left(5 \times 10^{4}\right)$ were plated out in 24-well plates and cultured overnight in normal growth medium. In initial experiments, two transfection reagents, siPortAmine and siPortLipid (Ambion), were tested under a range of conditions to optimise the protocol. The following protocol was used to generate the data described in the paper. Each well was transfected using $4 \mu \mathrm{l}$ of siPortAmine and $1 \mu \mathrm{l}$ of siRNA, to give final concentrations of $25 \mathrm{nM}$ of in vitrosynthesised siRNAs or up to $0.5 \mu \mathrm{g} \mathrm{ml}^{-1}$ of commercially synthesised siRNA. The siPortAmine was vortexed, added drop by drop to $45 \mu \mathrm{l}$ Opti-MEM1 (Invitrogen, Paisley, UK), vortexed and incubated for $30 \mathrm{~min}$ at room temperature. The diluted transfection reagent was added to the siRNA, mixed by pipetting and incubated for a further $30 \mathrm{~min}$. The growth medium was removed from the cells and replaced by $200 \mu \mathrm{l}$ Opti-MEM1 and then overlaid with the transfection reagent: siRNA complex, with rocking. The plates were incubated for $6 \mathrm{~h}$ at $37^{\circ} \mathrm{C}, 5 \% \mathrm{CO}_{2}$, after which $1 \mathrm{ml}$ growth medium was added and the plates were returned to the incubator.

\section{Real-time PCR}

RNA was extracted using RNABee (Biogenesis, Poole, UK) and complementary DNA (cDNA) synthesised using Superscript II and random hexamers as described previously (McWilliams et al, 1998). For each RNA, a cDNA-negative control (no RT or primer) was also synthesised. PCR for gastrin and the housekeeping gene was routinely carried out using $5 \mu \mathrm{l}$ of $1: 5 \mathrm{cDNA}$ and $20 \mu \mathrm{l}$ of reaction mix consisting of $1 \times$ reaction buffer, $1: 2000$ SybrGreen, $\mathrm{MgCl}_{2}$, dNTP mix, $1 \mu \mathrm{l}$ of primer and Hot GoldStar Taq (Eurogentec, Romsey, UK). The sequences of the primers used are shown in Table 2 . In addition, oligoadenylate synthase 1 (OAS), signal transducer and activator of transcription 1 (STAT1) and hypoxanthine guanine phosphoribosyl transferase (HPRT) genes were detected with Taqman primer sets (Applied Biosystems, Warrington, UK), using the same reaction mix as above but without SybrGreen.

Complementary DNAs were tested in triplicate and, in addition, cDNA-negative and 'no template' controls were analysed to ensure that the signal generated was derived from RNA and not from genomic DNA, primer-dimers or any of the cDNA or PCR reagents. The samples were run on a GeneAmp 5700 Sequence Detector Real-Time PCR machine (Applied Biosystems) using the following programme: $50^{\circ} \mathrm{C}$ for $2 \mathrm{~min}, 95^{\circ} \mathrm{C}$ for $10 \mathrm{~min}, 40$ cycles of $95^{\circ} \mathrm{C}$ for $15 \mathrm{~s}$ and $60^{\circ} \mathrm{C}$ for $1 \mathrm{~min}$ with products being detected

Table 2 Primers used for real-time PCR

\begin{tabular}{ll}
\hline Primer & Primer sequence $\left(\mathbf{5}^{\prime} \boldsymbol{\rightarrow} \mathbf{3}^{\prime}\right)$ \\
\hline Gastrin F & CCACACCTCGTGGCAGAC \\
Gastrin R & TCCATCCATCCATAGGCTTC \\
HPRT F & GACCAGTCAACAGGGGACAT \\
HPRT R & CGACCTTGACCATCTTTGGA \\
\hline
\end{tabular}


using SYBR Green or the fluorescent Taqman probe. The number of cycles required to reach a fluorescence threshold $\left(C_{\mathrm{t}}\right)$ during the exponential phase of amplification was determined for the test gene and for the housekeeping gene HPRT. Results are usually presented as relative gene expression in comparison to HPRT and a reference treatment using the $2^{-\Delta \Delta C t}$ method (Livak and Schmittgen, 2001), where

$$
\text { Relative gene expression }=\frac{2^{-\left[C t_{(\text {test gene })}-C t_{(H P R T)}\right]_{\text {test condition }}}}{2^{-\left[C t_{(\text {test gene })}-C t_{(H P R T)}\right]_{\text {reference condition }}}}
$$

To demonstrate differences in expression levels between different cell lines, data are shown using the $2^{-\Delta C t}$ equation where

$$
\left.2^{-\Delta C t}=2^{-\left[C t_{(\text {test gene })}-C t_{(H P R T)}\right]}\right]_{\text {test condition }}
$$

95\% confidence intervals for the mean gastrin expression were calculated based on the variation between the replicates for each sample and are indicated on each graph as error bars.

\section{Green fluorescent protein-tagged gastrin}

The complete gastrin coding sequence was amplified by PCR and cloned upstream of the green fluorescent protein (GFP) coding sequence in the plasmid pHRGFP-C (Stratagene, LaJolla, CA, USA), under the control of a CMV promoter, using the Bam $\mathrm{H} 1$ and XhoI restriction sites to create a plasmid pGasGFP. A complete Kozak sequence was incorporated into the forward primer, involving modification of the fourth nucleotide of the gastrin coding sequence from $\mathrm{C}$ to $\mathrm{G}$ (indicated in the primer sequence by underlining). The primers used were as follows:

Forward: CGCGGATCCGCCGCCGCCATGGAGCGACTGTG TGTG

Reverse: CCGCCGCTCGAGGCCGAAGTCCATCCATC

The unmodified pHRGFP-C plasmid was used as a vector control. As it lacks a Kozak sequence, no GFP expression was expected.

Dual transfection of plasmids and siRNAs was carried out by preparing the siRNA transfection mix as above but in half the volume, and preincubating $500 \mathrm{ng}$ of plasmid with $1 \mu \mathrm{l}$ Lipofectamine (Invitrogen, Paisley, UK) in $25 \mu \mathrm{l}$ serum-free medium. The two transfection reagents were then mixed immediately before addition to the cells.

\section{Antibody staining of cytospins}

Following transfection, cells were stored, fixed in $4 \%$ formalin overnight at $4{ }^{\circ} \mathrm{C}$, then washed twice in PBS after each of the following steps. Cytospins were made in a Shandon cytospin 4 using $10^{4}$ cells per slide. Cells were permeabilised by incubation with PBS containing $0.5 \%$ Triton $\mathrm{X}-100$ for $5 \mathrm{~min}$ at $4^{\circ} \mathrm{C}$. Nonspecific binding sites were blocked by a $30 \mathrm{~min}$ incubation at room temperature in PBS containing 3\% BSA and $1 \%$ glycine followed by PBS containing $10 \%$ normal swine serum (NSS). The primary antibody (rabbit anti-progastrin antibody, raised against amino acids 6-14 of progastrin conjugated to diphtheria toxin (DT) and purified against the BSA peptide conjugate; (Aphton Corp., Philadelphia, PA, USA) or control antibody (rabbit anti-DT) was added at a final concentration of $2.5 \mu \mathrm{g} \mathrm{ml}^{-1}$ in PBS containing $1 \%$ NSS and incubated overnight at $4{ }^{\circ} \mathrm{C}$. Binding of the antibody was detected using FITC-conjugated swine anti-rabbit immunoglobulin at a dilution of $1: 40$ (Dako, Ely, UK) and the cells were counterstained with Hoechst dye before examination under a fluorescent microscope.

\section{Growth assays}

Cell growth was measured using a standard methyl thiazoyl tetrazolium (MTT) assay (Watson et al, 1996). Briefly, following transfection with siRNAs, cells were grown overnight, then trypsinised, plated out into 96-well plates as replicates and incubated overnight in growth medium. The following day, the medium was replaced by RPMI 1640 culture medium supplemented with $1 \%$ serum or serum-free OptiMem1, and the cells were grown for a further $96 \mathrm{~h}$. In some experiments, OptiMem 1 was supplemented with $10 \mathrm{~nm}$ amidated gastrin (G17, Aphton, USA), $10 \mathrm{~nm}$ glycine-extended gastrin (Gly-G17, Aphton, USA) or the CCK2-R inhibitor, YM022 (James Black Foundation, Dulwich, UK) at $500 \mathrm{~nm}$. At intervals following transfection, the medium was replaced by fresh medium containing MTT at $1 \mathrm{mg} \mathrm{ml}^{-1}$ and incubated for $4 \mathrm{~h}$. The medium was removed, the incorporated MTT dissolved in DMSO and the OD at $550 \mathrm{~nm}$ was read.

\section{Caspase 3 inhibitor assay}

Cells undergoing apoptosis were identified by addition of a fluorescent caspase 3 inhibitor, FITC-DEVD-FMK (Calbiochem, Nottingham, UK), according to the manufacturer's instructions. Cells were harvested using trypsin-EDTA, resuspended in fresh medium and incubated with the inhibitor at the recommended final working concentration for $1 \mathrm{~h}$ at $37^{\circ} \mathrm{C}$. The cells were washed twice in the wash buffer provided, fixed in formalin and analysed on a BeckmanCoulter XL-MCL flow cytometer. The percentage of apoptotic cells was calculated, using untreated cells to define the baseline.

\section{Statistical analysis}

The significance of differences in gene expressing between cell lines was calculated using ANOVA with the Bonferroni multiple comparison test. The significance of differences between growth and rates of apoptosis in cells transfected with the gastrin siRNA and control siRNA was measured using the Student's $t$-test. Differences were considered to be significant if $P<0.05$.

\section{RESULTS}

\section{Localisation of suitable targets within gastrin}

A fluorescent TAMRA-labelled siRNA (tg8) was used to measure the efficiency of transfection. At $24 \mathrm{~h}$ after transfection, almost $100 \%$ of the cells were fluorescent with the signal associated with vesicle-like structures in the cytoplasm of the cells (Figure 1A). Small interfering RNAs directed against six different targets within gastrin and a control siRNA consisting of a scrambled version of the $\operatorname{tg} 5$ sequence were then used to transfect PAN-1 cells. Gene expression was measured $24 \mathrm{~h}$ after transfection by real-time PCR. Use of the most effective siRNAs, tg8 and tg9, resulted in a $93 \%$ decrease in gastrin gene expression relative to that in cells treated with the control siRNA and the housekeeping gene HPRT. The remaining siRNAs gave between 64 and $86 \%$ downregulation of the gastrin gene (Figure 1B). Tg8 was used for the remainder of the studies and synthesised commercially together with a scrambled control.

The gastrin siRNA is effective in a range of cell lines with different gastrin expression levels and is able to overcome transcriptional upregulation of the gastrin gene by EGF

The tg8 and scrtg8 siRNAs were used to transfect three additional cell lines, HCT116 (colorectal), C170HM2 (liver-metastasising variant of a colorectal cell line) and MGLVA1 (gastric). Basal gastrin gene expression in the four cell lines is shown in Figure 2A. Expression in HCT116 and C170HM2 cells was significantly higher (approximately $15 \times$ and $20 \times$, respectively; $P<0.001$ for both) than that in the PAN-1 and MGLVA1 cells. Nonetheless, in each of the cell lines, between 80 and 95\% downregulation of the gastrin gene was achieved in cells treated with gastrin siRNA (Figure 2B). In addition, the ability of the gastrin siRNA to overcome 

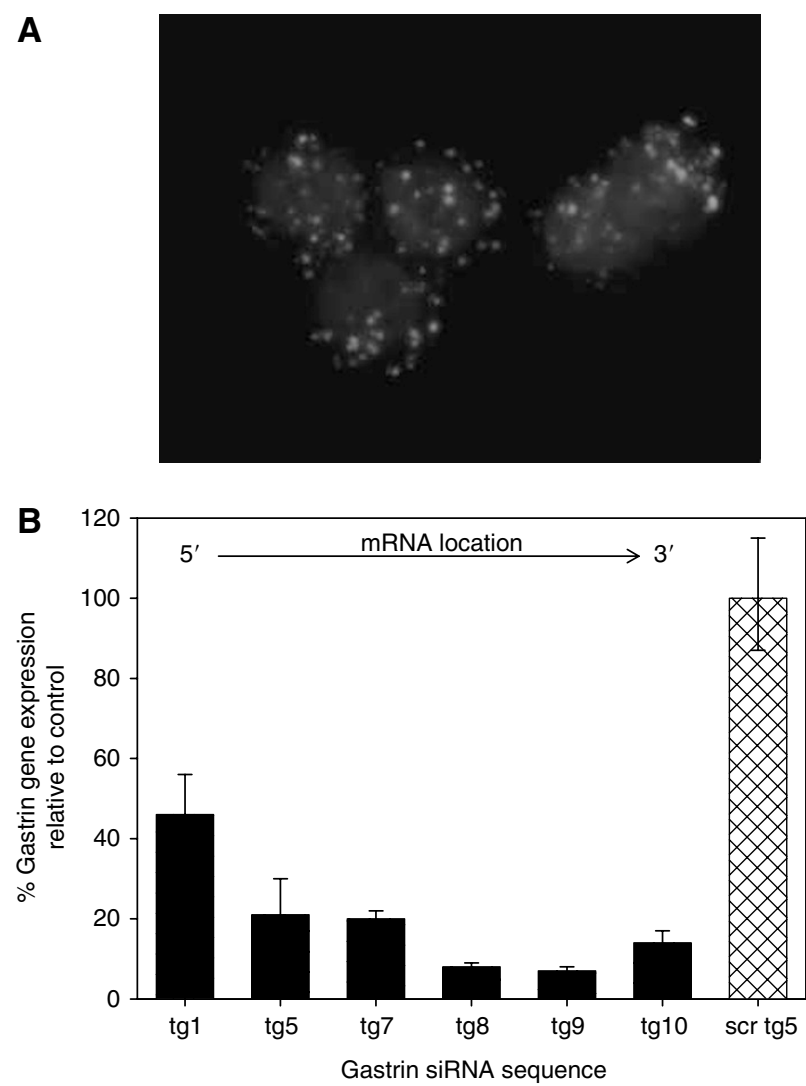

Figure I (A) siRNA transfection efficiency. PAN-I cells transfected with a TAMRA-labelled gastrin siRNA $24 \mathrm{~h}$ post-transfection $(\times 64$ magnification). The siRNA is associated with vesicle-like structures in the cytoplasm of the cells. (B) Expression of the gastrin gene in cells transfected with different gastrin siRNAs. PAN-I cells were transfected with six different gastrin siRNAs and gastrin expression was measured by real-time PCR. Expression relative to cells transfected with the scrambled control siRNA (scrtg5) and the housekeeping gene, HPRT, is shown. Error bars indicate the $95 \%$ confidence intervals. The relative position of each siRNA within the gastrin sequence is indicated.

upregulation of the gastrin gene by EGF was demonstrated in PAN1 cells (Figure 2C).

Downregulation of the gastrin gene by the siRNA is dosedependent and is maintained in vitro for more than 1 week

The tg8 and scrtg8 siRNAs were tested at a range of concentrations against HCT116 cells, one of the cell lines expressing the highest level of gastrin RNA. Downregulation of the gastrin gene was dosedependent (Figure 3), with $60 \%$ downregulation of the gene still achieved at the lowest effective dose of siRNA $(0.02 \mu \mathrm{g})$.

To investigate the duration of the effectiveness of the siRNA, PAN-1 cells were transfected with 0.5 or $0.05 \mu \mathrm{g}$ tg8 or scrtg8 siRNA, harvested at a range of time intervals following transfection and analysed for gene expression by real-time PCR. The effect of the tg8 siRNA was prolonged. At the highest concentration of siRNA used, $90 \%$ downregulation of the gene was maintained up to 7 days after transfection, dropping to $80 \%$ at day (d) 11 . At the lower concentration of siRNA, 85\% downregulation was maintained up to $\mathrm{d} 7$, but dropped to $53 \%$ by d11 (Figure 4 ). When a similar experiment (data not shown) was carried out with HCT116 cells, which have a higher basal level of gastrin expression, $60 \%$ downregulation of the gene was maintained up to d8 after transfection, provided that the higher concentration of siRNA was used. Thus, for the remainder of experiments, cells were transfected with $0.5 \mu \mathrm{g}$ siRNA per $5 \times 10^{4}$ cells.
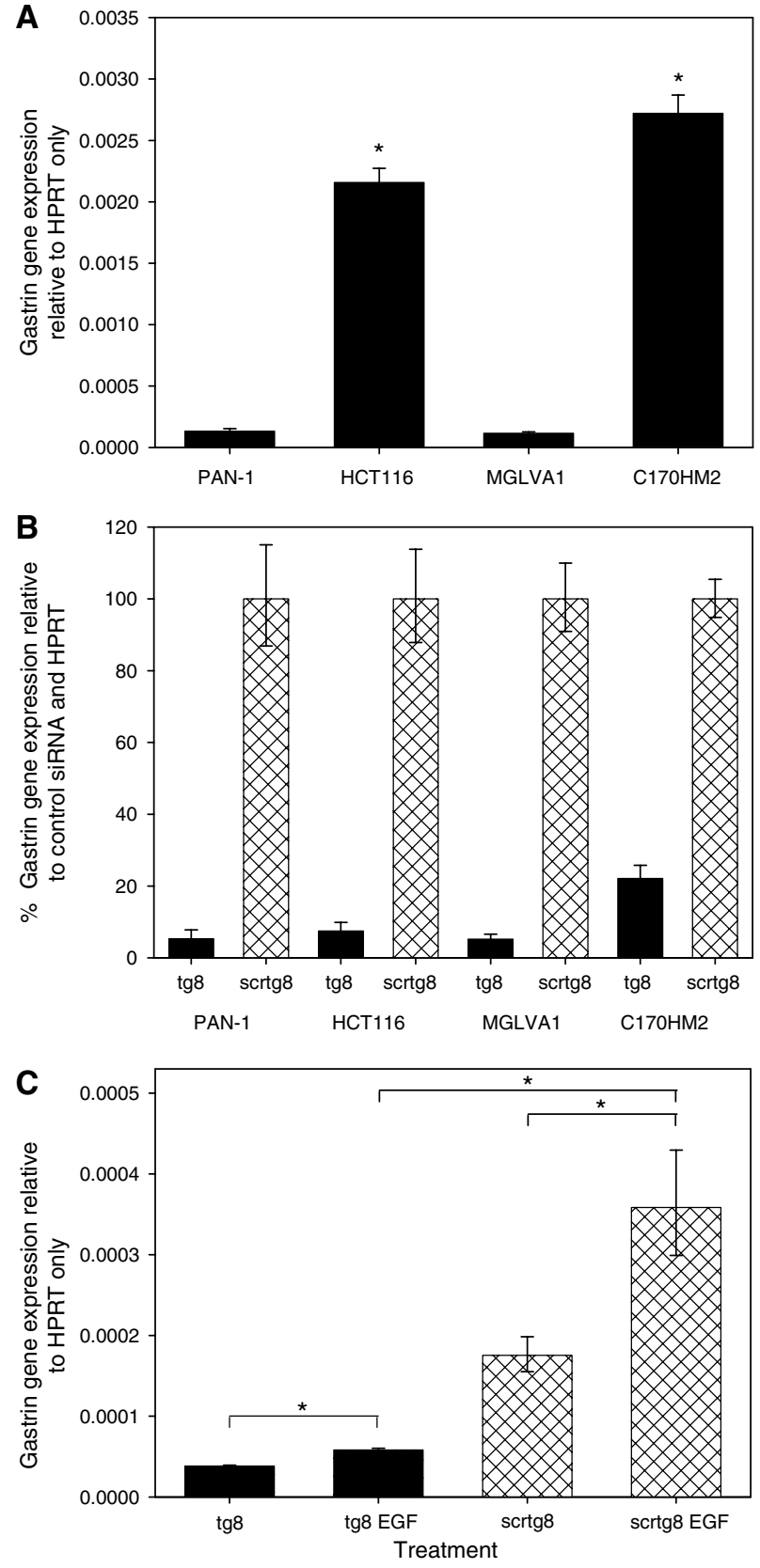

Figure 2 Effectiveness of gastrin siRNA in a range of $\mathrm{Gl}$ cancer cell lines. (A) Relative expression of gastrin in four cell lines (PAN-I, HCTII6, MGLVAI and $\mathrm{CI}$ 70HM2) is shown using the $2^{-\Delta C t}$ formula. A significant difference in expression compared with that in PAN-I $(P<0.00 \mathrm{I})$ is indicated by*. (B) The downregulation of gastrin gene expression in cells treated with the tg8 gastrin siRNA is shown relative to cells treated with the control scrtg8 siRNA using the $2^{-\Delta \Delta C t}$ formula. (C) Gene expression in PAN-I cells on d2 after transfection with gastrin (tg8) or control (scrtg8) siRNA and $24 \mathrm{~h}$ treatment with or without $10 \mu \mathrm{g} \mathrm{ml} l^{-1}$ EGF. Data are expressed relative to the housekeeping gene, HPRT. Significant differences $(P<0.00 \mathrm{I})$ are indicated by *. Error bars indicate the $95 \%$ confidence intervals.

The innate interferon response is not induced by the gastrin siRNA

To investigate whether transfection with gastrin siRNA results in induction of the innate interferon response, expression of STAT1 and OAS was measured in HCT116 cells transfected with tg8 or 

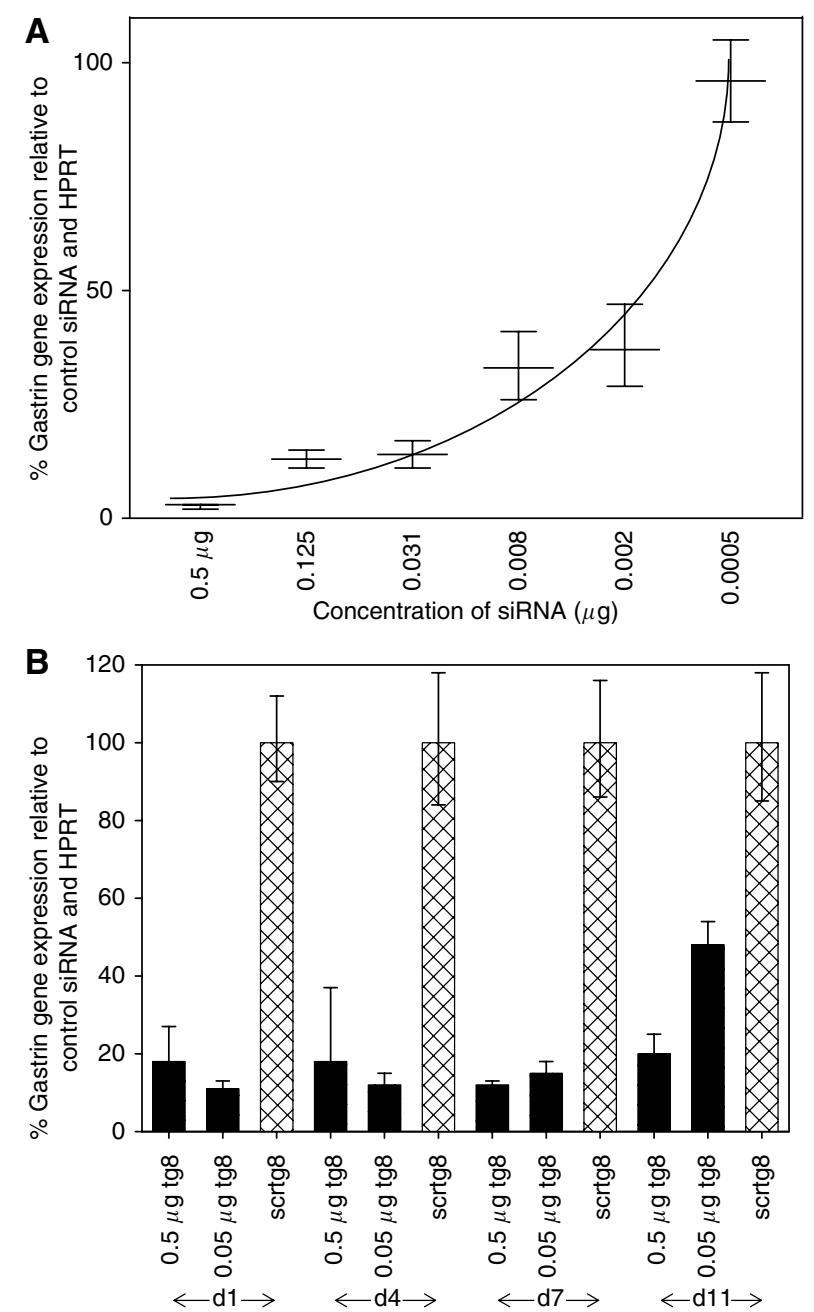

Figure 3 Concentration-dependent effects of the gastrin siRNA and longevity of downregulation of the gastrin gene. (A) Gastrin gene expression in HCTI I 6 cells transfected with a range of concentrations of gastrin (tg8) siRNA, expressed relative to the housekeeping gene, HPRT, and cells transfected with the same concentration of the control (scrtg8) siRNA. Error bars indicate the $95 \%$ confidence intervals. (B) Gene expression in PAN-I cells at dI, d4, d7 or d I I after transfection with 0.5 or $0.05 \mu$ g gastrin (tg8) or control (scrtg8) siRNA. Data are expressed relative to the control siRNA and the housekeeping gene, HPRT. Error bars indicate the $95 \%$ confidence intervals.

scrtg8 siRNA or mock-transfected cells treated with the transfection reagent but without siRNA. Whereas there was a significant difference comparing gastrin expression (Figure 4A) in tg8-treated cells with scrtg8- or mock-treated cells $(P=0.005)$, there was no significant difference in STAT1 (Figure 4B) or OAS (Figure 4C) expression $(P>0.28)$, suggesting that the innate interferon response is not induced by these siRNAs under these conditions.

\section{Downregulation of GFP-tagged and endogenous gastrin}

Owing to the complexity of the products arising from the gastrin gene and the low levels of gastrin protein produced by GI tumour cells, the effect of siRNA transfection on gastrin protein expression was initially analysed using HCT116 cells transfected with a GFPtagged gastrin gene. Four replicates of each cotransfection were carried out. One replicate for each treatment is shown in Figure 5A and $\mathrm{B}$. The average percentage of fluorescent cells following transfection with pGasGFP and the control siRNA was 19.8,
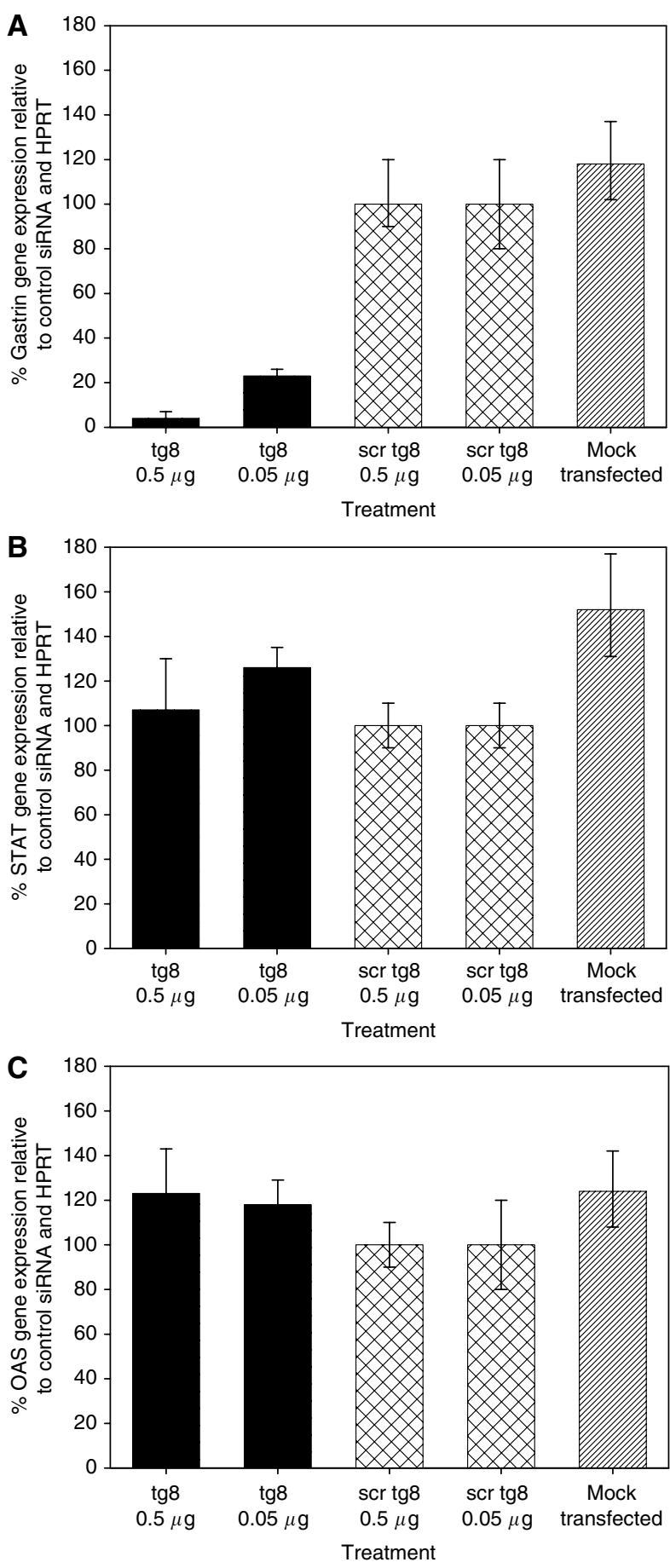

Figure 4 Absence of induction of the innate interferon response by gastrin siRNA. Gastrin, OAS and STATI gene expression in PAN-I cells treated with gastrin (tg8), control (scrtg8) siRNA or no siRNA (mock transfected). Error bars indicate the 95\% confidence intervals.

whereas only $4.8 \%$ of cells cotransfected with pGasGFP and the gastrin siRNA were positive. Thus, there was a significant reduction in the proportion of GFP-tagged gastrin-expressing cells within $24 \mathrm{~h}$ of treatment with the gastrin siRNA $(P<0.00001)$. However, a different pattern was seen when endogenous gastrin expression in siRNA-treated HCT116 cells was measured using a monoclonal antibody to amino acids 6-14 of progastrin. There was no apparent difference between tg8-and scrtg8-treated cells at early time points with the first detectable reduction in progastrin 


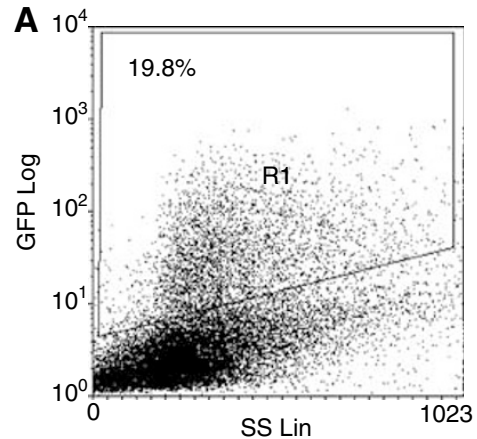

B

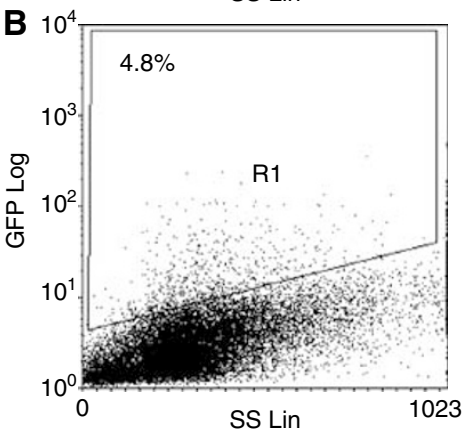

C
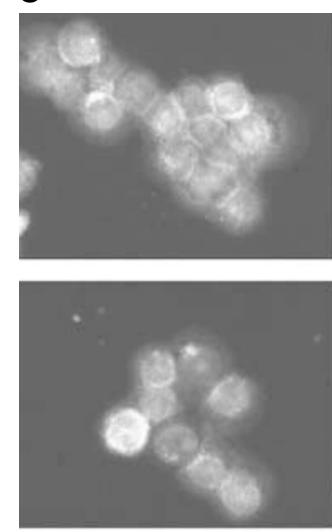

D
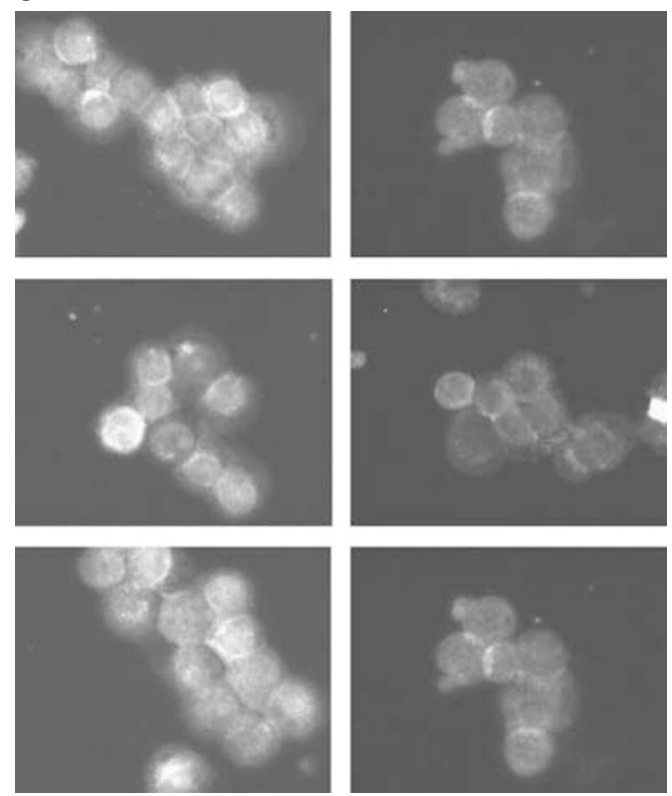

Figure 5 Downregulation of GFP-tagged and endogenous gastrin protein expression by gastrin siRNA. Cells transfected with (A) a GFP-tagged gastrin plasmid and treated with control scrtg8 siRNA or (B) gastrin tg8 siRNA are shown $24 \mathrm{~h}$ after transfection. The percentage of positive cells is indicated and there was a significant difference $(P<0.000 \mathrm{I})$ between the two treatments. Cells transfected with $(\mathbf{C})$ scrtg8 or $(\mathbf{D})$ gastrin tg8 siRNA were immunostained for endogenous gastrin expression using an anti-C-terminal antibody $72 \mathrm{~h}$ after transfection. Three fields are shown for each treatment.

staining observed at $\mathrm{d} 3$ after transfection (Figure $5 \mathrm{C}$ and $\mathrm{D}$ ), when there was still some residual staining of tg8-treated cells.

\section{Endogenous gastrin enhances survival of serum-starved GI cancer cells}

To measure the role of endogenous gastrin in cell survival following serum starvation, PAN-1 were cultured in medium supplemented with serum or serum-free medium (Figure 6A). Cell survival was assessed at a range of time points using an MTT assay. Under both sets of conditions, growth was lower in the gastrin siRNA-treated cells than in cells treated with the control siRNA. However, the effects reached significance only under serum-free conditions $(P<0.001)$, with $32 \%$ inhibition in serum-free medium compared with $13 \%$ (NS) in medium supplemented with serum at d8. Consistent with the delay in downregulation of endogenous gastrin protein expression following siRNA treatment, clear differences in cell survival were not apparent until approximately d6 following transfection.

As the gastrin siRNA reduces induction of the gastrin gene expression by EGF, cell survival was measured for each cell line in the presence or absence of $10 \mu \mathrm{g} \mathrm{ml}^{-1}$ EGF on $\mathrm{d} 6$ after transfection. The experiment was carried out on a minimum of three separate occasions and representative data for a single experiment for each cell line are shown in Figure 7. Although there was some variation between experiments, in all four cell lines there was reduced cell survival in the tg8-compared with the scrtg8-treated cells. Reduced thymidine incorporation was also observed in PAN-1 cells over a similar time frame (data not shown).

The effects were most marked in the C170HM2 cells, which expressed the highest level of gastrin. In the absence of EGF, there was a significant reduction in cell survival on each of the three occasions they were tested (\% cell survival of $63.1,75.0$ and 68.5 ), whereas in the remaining three cell lines, in the absence of EGF, significance was reached in only a proportion of separate experiments. Similar reductions in growth were seen in PAN-1 and $\mathrm{C} 170 \mathrm{HM} 2$ cells treated with two other gastrin siRNAs, $\operatorname{tg} 5$ and

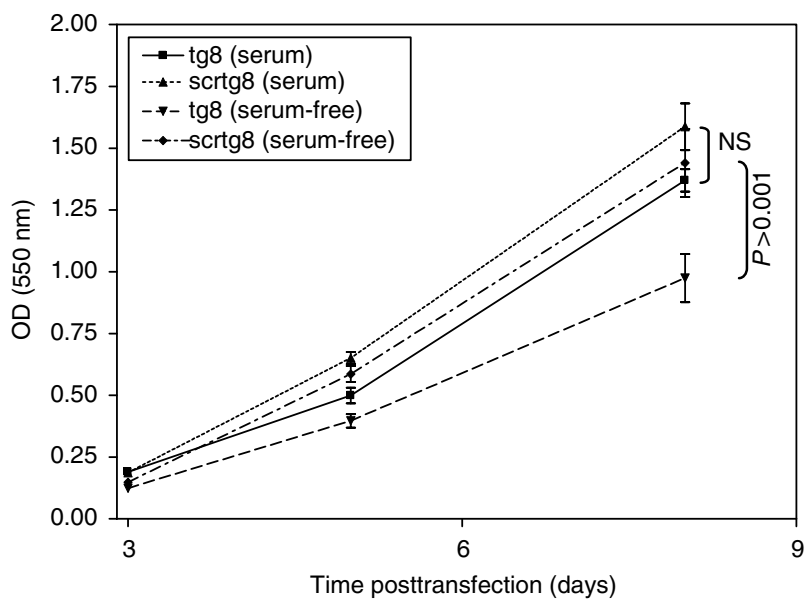

Figure 6 Survival of gastrin siRNA-transfected cells in the presence and absence of serum. Growth of PAN-I cells transfected with gastrin tg8 siRNA or the control scrtg8 siRNA in serum containing $10 \%$ or serum-free medium over 8 days following transfection. At $d 3$ and $d 6$, there was significantly lower survival in the tg8- treated cells than the scrtg8-treated cells $(P=0.004$ and 0.00 I for serum and no serum, respectively, at $\mathrm{d} 3$, and $P=0.06$ and 0.02 , respectively, at $d 6$ )

$\operatorname{tg} 7$ (data not shown), suggesting that this effect is specifically related to downregulation of the gastrin gene. In cells treated with EGF, a significant difference in growth was seen in every independent experiment and the percentage reduction in growth was greater.

\section{Downregulation of endogenous gastrin leads to increased apoptosis in GI cancer cells exposed to serum-free conditions}

As gastrin is thought to be anti-apoptotic as well as acting as a growth factor, the effect of downregulation of endogenous gastrin on 

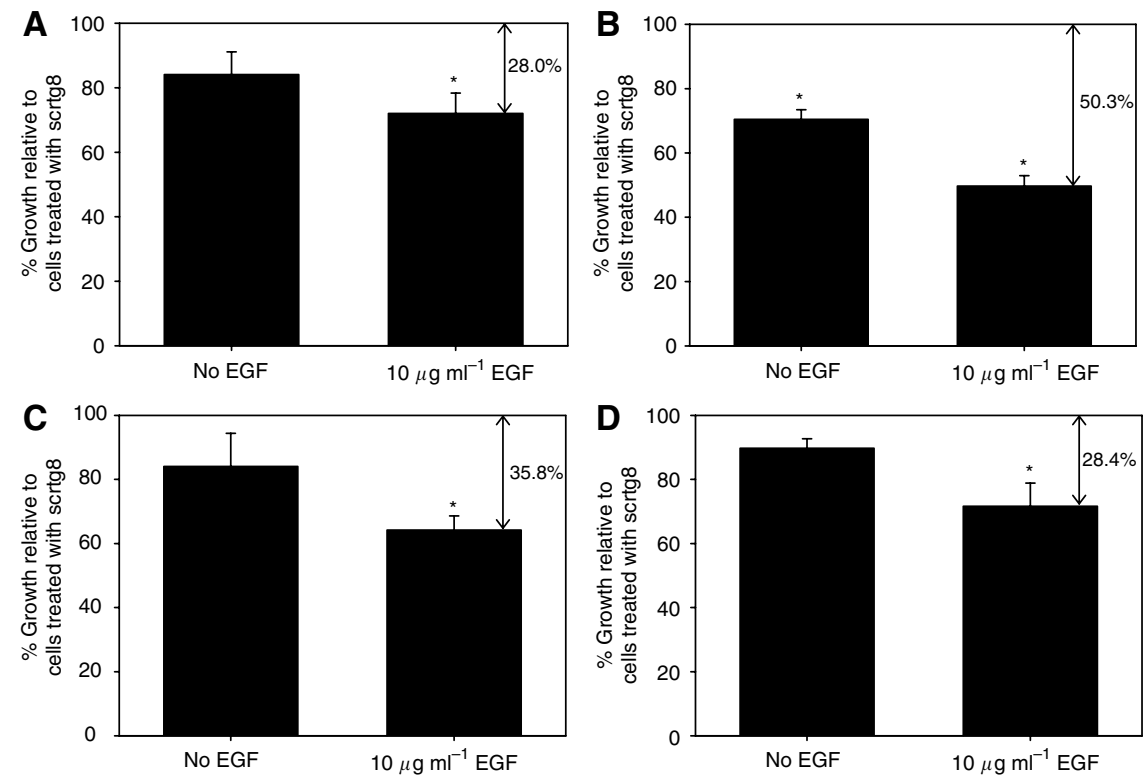

Figure 7 Survival of gastrin siRNA-transfected cancer cells. Survival of (A) PAN-I, (B) CI70HM2, (C) HCTII6 and (D) MGLVAI cells in serum-free medium with or without the addition of $10 \mu \mathrm{g} \mathrm{ml} \mathrm{l}^{-1}$ EGF following transfection with gastrin (tg8) or control (scrtg8) siRNAs. Growth of tg8-transfected cells is shown as a percentage of scrtg8-treated cells. Significant differences $(P<0.05)$ are indicated by *
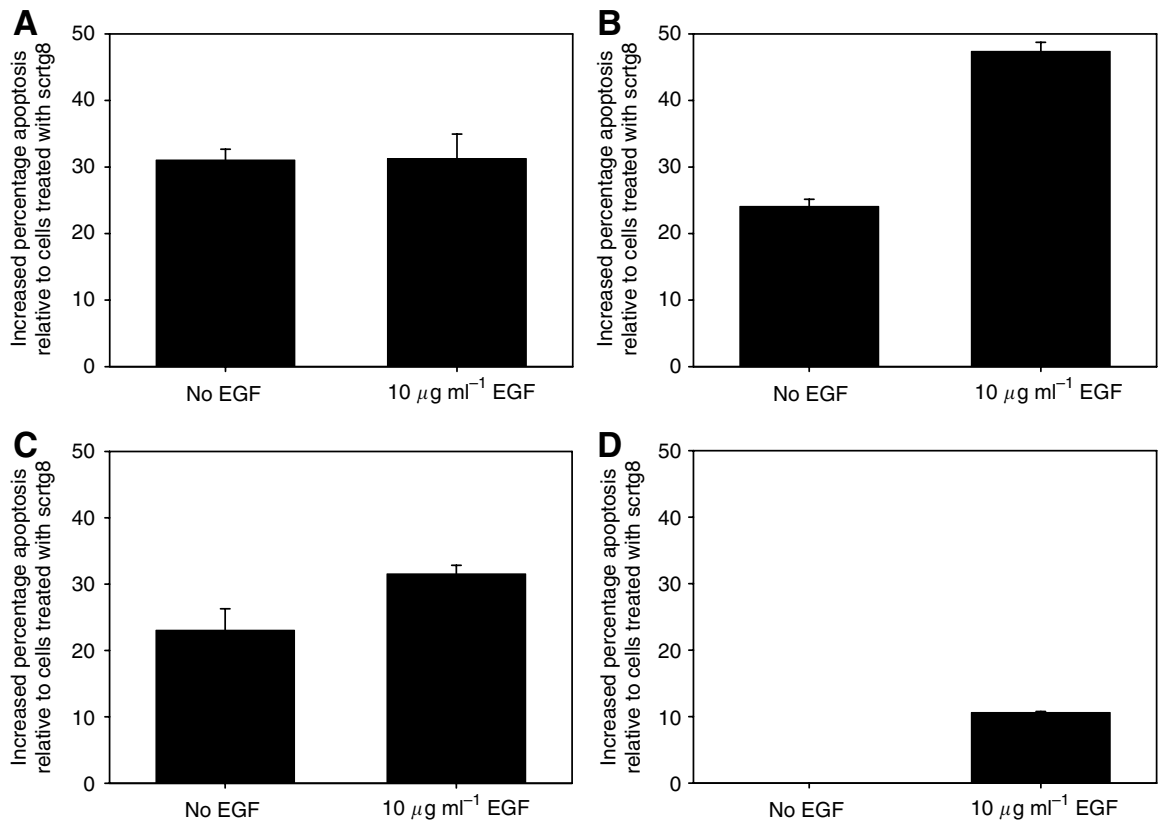

Figure 8 Apoptosis in gastrin siRNA-transfected cancer cells. Proportion of apoptotic cells at $\mathrm{d} 4$ following treatment with gastrin (tg8) or the contro (scrtg8) siRNA in the presence or absence of $10 \mu \mathrm{g} \mathrm{ml} l^{-1}$ EGF. Apoptosis is shown relative to scrtg8-treated cells. Significant differences $(P<0.05)$ are indicated by *. (A) PAN-I, (B) CI70HM2, (C) HCTII6 and (D) MGLVAI.

apoptosis in the four cell lines following exposure to serum-free conditions was also investigated. A fluorescent caspase 3 inhibitor was used to identify cells undergoing apoptosis, and the proportion of apoptotic cells, following transfection with the gastrin or control siRNA, was measured. Basal levels of apoptosis in the cell lines in serum-free medium varied between the different cells and in different experiments but was in the range of $10-30 \%$. The data at $\mathrm{d} 4$ following transfection are shown in Figure 8. There was increased apoptosis in the cells treated with the gastrin siRNA compared with the control siRNA, except in the MGLVA1 cells. In the absence of EGF, the difference was significant in the PAN-1 and C170HM2 cells. The effect was enhanced in the presence of EGF, with a significant difference seen additionally in the HCT116 cells. Significance was not reached in the MGLVA1 cells under either condition.

\section{Amidated gastrin and glycine-extended gastrin enhance growth of gastrin siRNA-treated cells}

To investigate the ability of exogenous gastrin to restore growth of cells treated with the gastrin siRNA, siRNA-transfected cells were treated with either amidated (G17) or glycine-extended gastrin (Gly-G17) at $10 \mathrm{nM}$. The growth of control siRNA-treated C170HM2s was increased by 23 and 56\%, following treatment with G17 and Gly-G17 respectively, and growth of the control 
siRNA-treated cells was increased by 75 and $88 \%$, respectively (Figure 9A). There was a significantly greater increase in growth in gastrin siRNA-transfected cells treated with G17 and GlyG17 than in control siRNA-treated cells $(P<0.001$ for both).

\section{Treatment of gastrin siRNA-treated cells with YM022} further reduced the survival of gastrin siRNA-transfected cells

The effect of the CCK2-R antagonist YM022 on survival of the gastrin siRNA-transfected cells was investigated in PAN-1 and C170HM2 cells. The antagonist had no significant effect on the growth of the control siRNA-treated cells but significantly reduced the survival of gastrin siRNA-transfected PAN-1 cells by $41 \%$ (Figure 9B, $P=0.003$ ). Survival of gastrin siRNA-transfected C170HM2 cells was reduced by $23 \%$ following treatment with YM022, but this did not reach significance.

\section{DISCUSSION}

Gastrin is involved in the establishment of a range of GI tumours. Here, using an siRNA that specifically targets gastrin, we show that endogenous gastrin plays a role in the survival of cell lines representing gastric, colorectal and pancreatic adenocarcinoma. Downregulation of gastrin resulted in reduced survival of cells exposed to serum-free conditions and was attributable at least in part to an increase in the rate of apoptosis in the gastrin siRNAtreated cells.

Initially, a number of siRNAs directed against potential targets within the gastrin gene were investigated for their effectiveness at mediating downregulation of the gastrin mRNA transcript. Interestingly, the most effective siRNAs were clustered towards the $3^{\prime}$ end of the transcript. This may be a result of easier access of the siRNA to its target due to reduced structural complexity in this region of the RNA as previously described (Luo and Chang, 2004; Yoshinari et al, 2004). The effects of the gastrin siRNA appear to be specific. The possibility of off-target effects (Scacheri et al, 2004) and induction of the interferon response (Sledz et al, 2003) has been suggested in the literature. A BLAST search was included in the design of the siRNAs used in this study and no matches to other genes were identified. Similar growth effects were seen with two additional gastrin siRNAs, and in all experiments a scrambled siRNA was used as a control for potential nonspecific effects due to the addition of siRNAs to the cell or transfection. In addition, we saw no induction of either OAS or STAT1 genes in response to the gastrin or control siRNA and the delay in the effects of the siRNA also argues against the involvement of the interferon response in the growth effects observed as this innate response is generally induced rapidly.

We were able to demonstrate rapid downregulation of plasmidencoded GFP-tagged gastrin peptides at the protein level within $24 \mathrm{~h}$ of treatment with the gastrin siRNA. However, there was an apparent delay of 2-3 days in downregulation of endogenous gastrin protein. Similar effects have been described previously for a number of genes (Wu et al, 2004) and may relate to slow rates of turnover of individual proteins within the cell or mechanisms that allow continued expression of protein from the small residual pool of mRNA; such mechanisms may not be active on recombinant vector-derived protein.

This apparent delay in downregulation of endogenous gastrin protein correlated with the time frame for measurement of the biological effects of downregulation of gastrin by the siRNA, which were delayed to approximately d5 following transfection. Downregulation of gastrin in the presence of serum resulted in a statistically significant reduction in cell survival, but the effect was small. However, in serum-free conditions, downregulation of gastrin reduced cell survival by up to $50 \%$ in the presence of
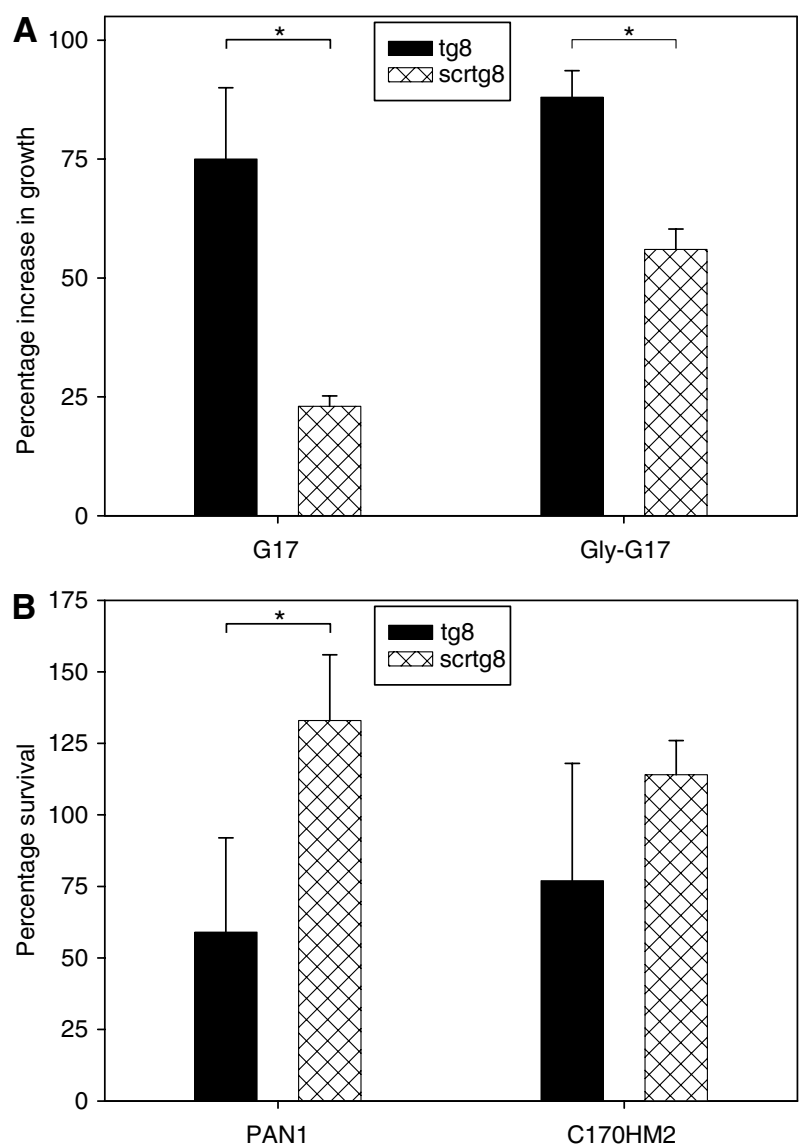

Figure 9 Growth of siRNA-transfected cells in the presence of gastrin peptides and a CCK2-R antagonist, (A) the growth of gastrin (tg8) or control (scrtg8) siRNA-transfected CI70HM2 cells treated with $10 \mathrm{nM}$ amidated (GI7) or glycine-extended (Gly-GI7) gastrin. The percentage increase in growth compared with transfected cells alone is indicated. There was a significant increase in the growth of peptide-treated gastrin siRNA-transfected cells compared with control siRNA-transfected cells ( $P<0.00$ I for both GI7 and GlyG I7, indicated by *). (B) The growth of gastrin (tg8) or control (scrtg8) siRNA-transfected Panl and CI70HM2 cells treated with $500 \mathrm{~nm}$ YM022 is shown. There was a significant further reduction in cell survival in tg8-transfected Pan I cells following treatment with YM022 $(41 \%, P=0.003)$ is shown. There was a $23 \%$ reduction in cell survival in $\mathrm{CI} 70 \mathrm{HM} 2$ cells but this did not reach significance.

EGF, the effect being most marked in the colorectal cell lines, especially C170HM2. This cell line has the highest constitutive levels of gastrin expression and may, therefore, be more dependent on gastrin for maintenance of the cell cycle.

The reduced survival of cells in which endogenous gastrin has been knocked down is in part due to an increase in apoptosis in these cells. The effects on apoptosis parallel the results of the cell survival assays, with the strongest effects seen in the colorectal cell lines, especially C170HM2, and with no significant effects on apoptosis seen in MGLVA1. A raised level of caspase 3 in cells treated with gastrin antisense and exposed to camptothecin has been reported previously in HCT116 cells (Wu et al, 2000).

Both gastrin and control siRNA-treated cells were responsive to amidated and glycine-extended gastrin. Interestingly, the gastrin siRNA-treated cells were more responsive, with not only greater percentage increase but also a higher final cell number than the control siRNA-treated cells treated with the peptides. This result further suggests that downregulation of the gastrin gene may lead to increased expression of the receptors for these peptides. 
This hypothesis is also supported by the data from the gastrin siRNA-transfected cells treated with the CCK2-R antagonist, YM022. The control siRNA-treated cells were unresponsive to YM022, suggesting that CCK2-R is not involved in the basal growth of these cells. This is in keeping with our findings that classical CCK2-R expression is undetectable in these cell lines by real-time PCR (data not shown) or using a nested PCR method that avoids potential artefacts associated with amplification of genomic DNA (Grabowska et al, 2004). It suggests that the gastrin-dependent cell survival, which is overcome by the gastrin siRNA, is not effected through the classical CCK2-R.

However, there was a decrease in the survival of the gastrin siRNA-treated cells in response to YM022. This could be explained by upregulation of one or more gastrin receptors that are inhibited by YM022, with either constitutive activity or with stimulation by residual endogenous gastrin. Thus, the percentage reduction in cell growth resulting from treatment with gastrin siRNA may be an underestimate of the role of gastrin in the basal growth of these cells if downregulation of gastrin leads to upregulation of alternative pathways that enhance cell growth.

The ability of the gastrin siRNAs to reduce survival of these GI cell lines is further evidence of the importance of autocrine and/or intracrine gastrin loops in the growth of these GI cancer cells. The effect of the gastrin siRNA is of a similar order of magnitude to that which has been described before when antibodies to gastrin peptides, receptor antagonists or antisense have been used to downregulate gastrin (Hoosein et al, 1988; Singh et al, 1996; Smith et al, 1996, 2004), and suggests that gastrin is only one of a number of factors contributing to survival of GI cancer cells. However, these data may also underestimate the role of endogenous gastrin. In the case of antibodies or receptor antagonists, they are effective against only a subset of gastrin isoforms. The siRNA described here is able to effectively downregulate gastrin at the RNA level and so should affect all products of the gastrin gene, but as downregulation of the protein is delayed and incomplete, there is only a narrow window in which the effect of gastrin knockdown can be measured. Further investigation of the mechanisms underlying gastrin translation and protein turnover would be beneficial. In addition, future studies will require systems to deliver the siRNA over a longer time frame, possibly through the use of plasmid-encoded small hairpin siRNAs or development of alternative approaches such as microRNAs that inhibit translation of the gastrin transcript. This will provide a model that will allow a more detailed study of the complex interactions between the different isoforms of gastrin and gastrin receptors expressed by these cells.

\section{REFERENCES}

Ahmed S, Budai B, Heredi-Szabo K, Farkas J, Toth G, Murphy RF, Lovas S (2004) High and low affinity receptors mediate growth effects of gastrin and gastrin-Gly on DLD-1 human colonic carcinoma cells. FEBS Lett 556: $199-203$

Aoki Y, Cioca DP, Oidaira H, Kamiya J, Kiyosawa K (2003) RNA interference may be more potent than antisense RNA in human cancer cell lines. Clin Exp Pharmacol Physiol 30: 96-102

Baba M, Itoh K, Tatsuta M (2004) Glycine-extended gastrin induces matrix metalloproteinase-1- and -3-mediated invasion of human colon cancer cells through type I collagen gel and Matrigel. Int J Cancer 111: 23-31

Baldwin GS (1995a) Binding of progastrin fragments to the $78 \mathrm{kDa}$ gastrinbinding protein. FEBS Lett 359: $97-100$

Baldwin GS (1995b) The role of gastrin and cholecystokinin in normal and neoplastic gastrointestinal growth. J Gastroenterol Hepatol 10: 215-232

Baldwin GS, Hollande F, Yang Z, Karelina Y, Paterson A, Strang R, Fourmy D, Neumann G, Shulkes A (2001) Biologically active recombinant human progastrin(6-80) contains a tightly bound calcium ion. J Biol Chem 276: $7791-7796$

Clarke PA, Dickson JH, Harris JC, Grabowska A, Watson SA (2006) Gastrin enhances the angiogenic potential of endothelial cells via modulation of heparin binding epidermal-like growth factor. Cancer Res 66: 3504-3512

Dockray GJ, Varro A, Dimaline R, Wang T (2001) The gastrins: their production and biological activities. Annu Rev Physiol 63: 119-139

Elbashir SM, Harborth J, Lendeckel W, Yalcin A, Weber K, Tuschl T (2001a) Duplexes of 21-nucleotide RNAs mediate RNA interference in cultured mammalian cells. Nature 411: 494-498

Elbashir SM, Lendeckel W, Tuschl T (2001b) RNA interference is mediated by 21- and 22-nucleotide RNAs. Genes Dev 15: 188-200

Finley GG, Koski RA, Melhem MF, Pipas JM, Meisler AI (1993) Expression of the gastrin gene in the normal human colon and colorectal adenocarcinoma. Cancer Res 53: 2919-2926

Goetze JP, Nielsen FC, Burcharth F, Rehfeld JF (2000) Closing the gastrin loop in pancreatic carcinoma: coexpression of gastrin and its receptor in solid human pancreatic adenocarcinoma. Cancer 88: 2487-2494

Grabowska AM, Clarke P, Watson SA (2004) Development of a specific RT - PCR method to detect expression of CCK-2 receptor isoforms. In 15th International Symposium on Regulatory Peptides. Regulatory Peptides 122: 47

Harris JC, Clarke PA, Awan A, Jankowski J, Watson SA (2004a) An antiapoptotic role for gastrin and the gastrin/CCK-2 receptor in Barrett's esophagus. Cancer Res 64: 1915-1919

Harris JC, Gilliam AD, McKenzie AJ, Evans SA, Grabowska AM, Clarke PA, McWilliams DF, Watson SA (2004b) The biological and therapeutic

importance of gastrin gene expression in pancreatic adenocarcinomas. Cancer Res 64: 5624-5631

Henwood M, Clarke PA, Smith AM, Watson SA (2001) Expression of gastrin in developing gastric adenocarcinoma. Br J Surg 88: $564-568$

Hoosein NM, Kiener PA, Curry RC, Rovati LC, McGilbra DK, Brattain MG (1988) Antiproliferative effects of gastrin receptor antagonists and antibodies to gastrin on human colon carcinoma cell lines. Cancer Res 48: $7179-7183$

Howell GM, Ziober BL, Humphrey LE, Willson JK, Sun L, Lynch M, Brattain MG (1995) Regulation of autocrine gastrin expression by the TGF alpha autocrine loop. J Cell Physiol 162: 256-265

Kermorgant S, Lehy T (2001) Glycine-extended gastrin promotes the invasiveness of human colon cancer cells. Biochem Biophys Res Commun 285: $136-141$

Konturek PC, Kania J, Kukharsky V, Ocker S, Hahn EG, Konturek SJ (2003) Influence of gastrin on the expression of cyclooxygenase-2, hepatocyte growth factor and apoptosis-related proteins in gastric epithelial cells. J Physiol Pharmacol 54: 17-32

Livak KJ, Schmittgen TD (2001) Analysis of relative gene expression data using real-time quantitative PCR and the 2(-Delta Delta $C(\mathrm{~T}))$ method. Methods 25: $402-408$

Luo KQ, Chang DC (2004) The gene-silencing efficiency of siRNA is strongly dependent on the local structure of mRNA at the targeted region. Biochem Biophys Res Commun 318: 303-310

McWilliams DF, Watson SA, Crosbee DM, Michaeli D, Seth R (1998) Coexpression of gastrin and gastrin receptors (CCK-B and delta CCK-B) in gastrointestinal tumour cell lines. Gut 42: 795-798

Ramamoorthy S, Stepan V, Todisco A (2004) Intracellular mechanisms mediating the anti-apoptotic action of gastrin. Biochem Biophys Res Commun 323: $44-48$

Rehfeld JF, Bundgaard JR, Goetze JP, Friis-Hansen L, Hilsted L, Johnsen AH (2004) Naming progastrin-derived peptides. Regul Pept 120: $177-183$

Scacheri PC, Rozenblatt-Rosen O, Caplen NJ, Wolfsberg TG, Umayam L, Lee JC, Hughes CM, Shanmugam KS, Bhattacharjee A, Meyerson M, Collins FS (2004) Short interfering RNAs can induce unexpected and divergent changes in the levels of untargeted proteins in mammalian cells. Proc Natl Acad Sci USA 101: 1892-1897

Seva C, Dickinson CJ, Yamada T (1994) Growth-promoting effects of glycine-extended progastrin. Science 265: 410-412

Shulkes A, Baldwin GS (1997) Biology of gut cholecystokinin and gastrin receptors. Clin Exp Pharmacol Physiol 24: 209-216

Singh P, Owlia A, Espeijo R, Dai B (1995) Novel gastrin receptors mediate mitogenic effects of gastrin and processing intermediates of gastrin on 
Swiss 3T3 fibroblasts. Absence of detectable cholecystokinin (CCK)-A and CCK-B receptors. J Biol Chem 270: 8429-8438

Singh P, Owlia A, Varro A, Dai B, Rajaraman S, Wood T (1996) Gastrin gene expression is required for the proliferation and tumorigenicity of human colon cancer cells. Cancer Res 56: 4111-4115

Sledz CA, Holko M, de Veer MJ, Silverman RH, Williams BR (2003) Activation of the interferon system by short-interfering RNAs. Nat Cell Biol 5: 834-839

Smith AM, Watson SA (2000) Gastrin and gastrin receptor activation: an early event in the adenoma-carcinoma sequence. Gut 47: $820-824$

Smith JP, Shih A, Wu Y, McLaughlin PJ, Zagon IS (1996) Gastrin regulates growth of human pancreatic cancer in a tonic and autocrine fashion. Am J Physiol 270: R1078-R1084

Smith JP, Verderame MF, Ballard EN, Zagon IS (2004) Functional significance of gastrin gene expression in human cancer cells. Regul Pept 117: $167-173$

Smith JP, Verderame MF, McLaughlin P, Martenis M, Ballard E, Zagon IS (2002) Characterization of the CCK-C (cancer) receptor in human pancreatic cancer. Int J Mol Med 10: 689-694

Stepan VM, Sawada M, Todisco A, Dickinson CJ (1999) Glycine-extended gastrin exerts growth-promoting effects on human colon cancer cells. Mol Med 5: 147-159

Todisco A, Ramamoorthy S, Witham T, Pausawasdi N, Srinivasan S, Dickinson CJ, Askari FK, Krametter D (2001) Molecular mechanisms for the antiapoptotic action of gastrin. Am J Physiol Gastrointest Liver Physiol 280: G298-G307
Watson S, Durrant L, Morris D (1989) Gastrin: growth enhancing effects on human gastric and colonic tumour cells. $\mathrm{Br}$ J Cancer 59: 554-558

Watson SA, Durrant LG, Morris DL (1990) The effect of the E2 prostaglandin enprostil, and the somatostatin analogue SMS 201 995, on the growth of a human gastric cell line, MKN45G. Int J Cancer 45: 90 - 94

Watson SA, Michaeli D, Grimes S, Morris TM, Robinson G, Varro A, Justin TA, Hardcastle JD (1996) Gastrimmune raises antibodies that neutralize amidated and glycine-extended gastrin-17 and inhibit the growth of colon cancer. Cancer Res 56: 880-885

Watson SA, Morris TM, Crosbee DM, Hardcastle JD (1993) A hepatic invasive human colorectal xenograft model. Eur J Cancer 29A: 1740-1745

Watson SA, Robinson KE, McWilliams D, Michaeli D, Smith AM, Robinson G (2000) Transforming growth factor-alpha-mediated growth pathways in human gastro-intestinal cell lines in relation to the gastrin autocrine pathway. Int J Cancer 87: 20-28

Watson SA, Smith AM (2001) Hypergastrinemia promotes adenoma progression in the APC(Min-/+) mouse model of familial adenomatous polyposis. Cancer Res 61: 625-631

Wu H, Rao GN, Dai B, Singh P (2000) Autocrine gastrins in colon cancer cells up-regulate cytochrome $c$ oxidase $\mathrm{Vb}$ and down-regulate efflux of cytochrome $c$ and activation of caspase-3. J Biol Chem 275: 32491-32498

Wu W, Hodges E, Redelius J, Hoog C (2004) A novel approach for evaluating the efficiency of siRNAs on protein levels in cultured cells. Nucleic Acids Res 32: e17

Yoshinari K, Miyagishi M, Taira K (2004) Effects on RNAi of the tight structure, sequence and position of the targeted region. Nucleic Acids Res 32: $691-699$ 